\title{
A POSTERIORI ERROR ESTIMATION OF FINITE ELEMENT APPROXIMATIONS OF POINTWISE STATE CONSTRAINED DISTRIBUTED CONTROL PROBLEMS*
}

\author{
RONALD H.W. HOPPE AND MICHAEL KIEWEG ${ }^{\dagger}$
}

\begin{abstract}
We provide an a posteriori error analysis of finite element approximations of pointwise state constrained distributed optimal control problems for second order elliptic boundary value problems. In particular, we derive a residual-type a posteriori error estimator and prove its efficiency and reliability up to oscillations in the data of the problem and a consistency error term. In contrast to the case of pointwise control constraints, the analysis is more complicated, since the multipliers associated with the state constraints live in measure spaces. The analysis essentially makes use of appropriate regularizations of the multipliers both in the continuous and in the discrete regime. Numerical examples are given to illustrate the performance of the error estimator.
\end{abstract}

Key words. optimal control, state constraints, adaptive finite elements, a posteriori error analysis, efficiency and reliability

AMS subject classifications. 49M15, 65K10, 65N30, 65N50

1. Introduction. The theory and application of adaptive finite element methods for the efficient numerical solution of boundary and initial-boundary value problems for partial differential equations (PDEs) has reached some state of maturity as documented by a series of monographs. There exist several concepts including residual and hierarchical type estimators, error estimators that are based on local averaging, the so-called goal oriented dual weighted approach, and functional type error majorants (cf. $[1,2,3,12,30,32]$ and the references therein).

On the other hand, as far as the development of adaptive finite element schemes for optimal control problems for PDEs is concerned, much less work has been done. The goal oriented dual weighted approach has been applied to unconstrained problems in $[3,4]$. Residual-type a posteriori error estimators for control constrained problems have been derived and analyzed in [14, 15, 18, 22, 24, 26, 27], whereas the theory of functional type error majorants has been investigated for control constrained elliptic problems in [16].

As opposed to the control constrained case, the difficulty associated with pointwise state constrained optimal control problems is due to the fact that the Lagrange multiplier for the state constraints lives in a measure space (see, e.g., [7, 8, 20,31]). Finite difference and finite element approximations of such problems have been studied both with regard to a priori error estimates $[9,10,13]$ as well as with respect to the efficient iterative solution of the discretized problems by primal-dual active set strategies and interior-point methods [5, 6, 19, 21, 23].

However, an a posteriori error analysis of adaptive finite element approximations of pointwise state constrained control problems has not yet been provided. In this paper, we attempt to close this gap by the development, analysis and implementation of a residual type a posteriori error estimator. The paper is organized as follows:

In section 2, as a model problem we consider a distributed optimal control problem

*The authors acknowledge support by the NSF under Grant No. DMS-0411403 and Grant No. DMS-0511611 as well as by the DFG within the Priority Program SPP 1253'PDE Constrained Optimization'.

${ }^{\dagger}$ Department of Mathematics, University of Houston, Houston, TX 77204 (rohop@math.uh.edu) and Institute of Mathematics, University of Augsburg, D-86159 Augsburg, Germany (hoppe@math.uni-augsburg.de). 
for a two-dimensional, second order elliptic PDE with a quadratic objective functional and unilateral constraints on the state variable. The optimality conditions are stated in terms of the state, the adjoint state, the control, and a Lagrangian multiplier for the state constraints which lives in the space of Radon measures. We further introduce a regularized multiplier and a modified adjoint state which will play an essential role in the error analysis.

In section 3, we describe the finite element discretization of the control problem with respect to a family of shape regular simplicial triangulations of the computational domain using continuous, piecewise linear finite elements for the state, the control, the adjoint and the modified adjoint state, and the regularized multiplier, whereas the multiplier itself is approximated by Dirac delta functionals associated with the nodal points of the triangulations.

In section 4, we present the residual-type a posteriori error estimator for the global discretization errors in the state, the adjoint state and the control. Data oscillations are considered as well, since they essentially enter the error analysis which is the subject of the subsequent sections 5 and 6 .

In particular, in section 5 we prove reliability of the error estimator, i.e., we prove that it provides an upper bound for the global discretization errors up to data oscillations. Section 6 deals with the efficiency of the estimator by showing that, modulo data oscillations, the error estimator also gives rise to a lower bound for the discretization errors.

Finally, section 7 is devoted to a detailed documentation of numerical results for two representative test examples in terms of the convergence history of the adaptive finite element process including visualizations of the adaptively generated simplicial triangulations.

2. The state constrained distributed control problem. Let $\Omega \subset \mathbb{R}^{2}$ be a bounded, polygonal domain with boundary $\Gamma:=\Gamma_{D} \cup \Gamma_{N}, \Gamma_{D} \cap \Gamma_{N}=\emptyset$. We use standard notation from Lebesgue and Sobolev space theory and refer to $W^{k, p}(\Omega), k \in$ $\mathbb{N}, 1<p<\infty$, as the Sobolev spaces with norms $\|\cdot\|_{k, p, \Omega}$. Note that for $k=0$ we obtain the Lebesgue space $L^{p}(\Omega)$. In case $p=2$, we refer to $(\cdot, \cdot)_{0, \Omega}$ as the inner product of the Hilbert space $L^{2}(\Omega)$, and we will write $H^{k}(\Omega)$ instead of $W^{k, 2}(\Omega)$ and $\|\cdot\|_{k, \Omega}$ instead of $\|\cdot\|_{k, 2, \Omega}$. For $k \geq 1$, we further refer to $\left.\right|_{k, p, \Omega}$ as the associated seminorm on $W^{k, p}(\Omega)$ which actually is a norm on $W_{0}^{k, p}(\Omega):=\{v \in$ $\left.W^{k, p}(\Omega)\left|\left(D^{\alpha} v\right)\right|_{\Gamma}=0,|\alpha| \leq k-1\right\}$. If we consider functions in $W^{k, p}(D), D \subset \Omega$, we will write $\|\cdot\|_{k, p, D}$ and $|\cdot|_{k, p, D}$, respectively. We recall that for $q$ conjugate to $p$ in the sense that $1 / p+1 / q=1$, the space $W^{-k, q}(\Omega)$ is dual to $W^{k, p}(\Omega)$. Finally, we denote by $C(\bar{\Omega})$ the Banach space of continuous functions on $\bar{\Omega}$. Its dual $\mathcal{M}(\Omega)=C(\bar{\Omega})^{*}$ is the space of Radon measures on $\Omega$ with $\langle\cdot, \cdot\rangle$ standing for the associated dual pairing. We refer to $C_{+}(\bar{\Omega})$ and $\mathcal{M}_{+}(\Omega)$ as the positive cones of $C(\bar{\Omega})$ and $\mathcal{M}(\Omega)$. In particular, $\sigma \in \mathcal{M}_{+}(\Omega)$ iff $\langle\sigma, v\rangle \geq 0$ for all $v \in C_{+}(\bar{\Omega})$.

For given $c \in \mathbb{R}_{+}$, we refer to $A: V \rightarrow H^{-1}(\Omega), V:=\left\{v \in H^{1}(\Omega)|v|_{\Gamma_{D}}=0\right\}$, as the linear second order elliptic differential operator

$$
A y:=-\Delta y+c y \quad, \quad y \in V
$$

and to $a(\cdot, \cdot): V \times V \rightarrow \mathbb{R}$ with $a(y, v):=\int_{\Omega}(\nabla y \cdot \nabla v+c y v) d x$ as the associated bilinear form. We assume $c>0$ or meas $\left(\Gamma_{D}\right) \neq 0$. In particular, this assures that $A$ is bounded and $V$-elliptic, i.e., there exist constants $C>0$ and $\gamma>0$ such that

$$
|a(y, v)| \leq C\|y\|_{1, \Omega}\|v\|_{1, \Omega} \quad, \quad a(y, y) \geq \gamma\|y\|_{1, \Omega}^{2} .
$$


We further assume that $\Omega$ is such that for all $u \in L^{2}(\Omega)$ the solution of the elliptic boundary value problem

$$
A y=u \text { in } \Omega, y=0 \text { on } \Gamma_{D}
$$

satisfies $y \in V \cap W^{1, r}(\Omega)$ for some $r>2$. We note that this allows nonconvex domains, e.g., such with reentrant corners (cf. [17]). According to the Sobolev imbedding theorem we have $y \in C(\bar{\Omega})$.

Now, given a desired state $y^{d} \in L^{2}(\Omega)$, a shift control $u^{d} \in L^{2}(\Omega)$, a regularization parameter $\alpha>0$ and a function $\psi \in W^{1, r}(\Omega)$ satisfying $\left.\psi\right|_{\Gamma_{D}}>0$, we consider the objective functional

$$
J(y, u):=\frac{1}{2}\left\|y-y^{d}\right\|_{0, \Omega}^{2}+\frac{\alpha}{2}\left\|u-u^{d}\right\|_{0, \Omega}^{2}
$$

and the associated state constrained distributed optimal control problem: Find $(y, u) \in$ $V \times L^{2}(\Omega)$ such that

$$
\inf _{y, u} J(y, u)
$$

subject to the constraints

$$
\begin{aligned}
& A y=u \text { in } \Omega, y=0 \text { on } \Gamma_{D}, \\
& I y \in K:=\{v \in C(\bar{\Omega}) \mid v(x) \leq \psi(x), x \in \bar{\Omega}\},
\end{aligned}
$$

where $I$ stands for the embedding operator $I: W^{1, r}(\Omega) \hookrightarrow C(\bar{\Omega})$. Since the solution $y=y(u)$ of $(2.1)$ lives in $V \cap C(\bar{\Omega})$, we define $G: L^{2}(\Omega) \rightarrow C(\bar{\Omega})$ as the control-to-state map which assigns to $u \in L^{2}(\Omega)$ the unique solution $y=y(u)$ of (2.1). We note that the control-to-state map $G$ is a bounded linear operator.

We assume that the following Slater condition is satisfied

$$
\text { There exists } v_{0} \in L^{2}(\Omega) \text { such that } G v_{0} \in \operatorname{int}(K) \text {. }
$$

We note that the assumption $\left.\psi\right|_{\Gamma_{D}}$ is necessary for (2.5) to hold true.

Substituting the state $y=y(u)$ by $y(u)=G u$ leads to the reduced objective functional

$$
J_{\text {red }}(u):=\frac{1}{2}\left\|G u-y^{d}\right\|_{0, \Omega}^{2}+\frac{\alpha}{2}\left\|u-u^{d}\right\|_{0, \Omega}^{2},
$$

which allows to reformulate the optimal control problem (2.2)-(2.4) according to

$$
\inf _{u \in U_{a d}} J_{r e d}(u), U_{a d}:=\left\{v \in L^{2}(\Omega) \mid(G v)(x) \leq \psi(x), x \in \bar{\Omega}\right\} .
$$

Since $J_{\text {red }}$ is lower semicontinuous, strictly convex and coercive, and the admissible control set $U_{a d}$ is closed and convex, the optimal control problem has a unique solution. The optimality conditions for the optimal solution $(y, u) \in V \cap L^{2}(\Omega)$ are given as follows.

THEOREM 2.1. The optimal solution $(y, u) \in K \times L^{2}(\Omega)$ of (2.2)-(2.4) is characterized by the existence of an adjoint state $p \in V_{s}$, where $V_{s}:=\left\{v \in W^{1, s}(\Omega)|v|_{\Gamma_{D}}=\right.$ 
$0\}$ and $s$ is conjugate to $r$, and a multiplier $\sigma \in \mathcal{M}_{+}(\Omega)$ such that

$$
\begin{aligned}
(\nabla y, \nabla v)_{0, \Omega}+(c y, v)_{0, \Omega} & =(u, v)_{0, \Omega}, v \in V, \\
(\nabla p, \nabla w)_{0, \Omega}+(c p, w)_{0, \Omega} & =\left(y-y^{d}, w\right)_{0, \Omega}+\langle\sigma, w\rangle, w \in V_{s}, \\
p+\alpha\left(u-u^{d}\right) & =0 \\
\langle\sigma, y-\psi\rangle & =0 .
\end{aligned}
$$

Proof. The proof follows the lines of [8]. Since there are stronger regularity assumptions in [8], it will be presented here. Denoting by $I_{K}$ the indicator function of the constraint set $K$, the reduced problem (2.6) can be written in the formally unconstrained form

$$
\inf _{v \in L^{2}(\Omega)} \hat{J}(v):=J_{\text {red }}(v)+\left(I_{K} \circ G\right)(v) .
$$

The optimal solution $u \in L^{2}(\Omega)$ satisfies $0 \in \partial \hat{J}(u)$, where $\partial \hat{J}(u)$ stands for the subdifferential of $\hat{J}$ at $u$. Due to the Slater condition, subdifferential calculus tells us $\partial\left(I_{K} \circ G\right)(u)=G^{*} \circ \partial I_{K}(G u)$ and hence, (2.11) results in

$$
0 \in J_{\text {red }}^{\prime}(u)+G^{*} \circ \partial I_{K}(G u) .
$$

Consequently, there exists $\sigma \in \partial I_{K}(G u)$ such that

$$
\left(y(u)-y^{d}, y(v)\right)_{0, \Omega}+\alpha\left(u-u^{d}, v\right)_{0, \Omega}+\left(G^{*} \sigma, v\right)_{0, \Omega}=0, v \in L^{2}(\Omega) .
$$

We define

$$
\bar{\sigma}:=G^{*} \sigma
$$

as a regularization of $\sigma \in \mathcal{M}(\Omega)$ and obtain from Theorem 4 in [8] that $\bar{\sigma} \in V_{s}$ with $1<s<2$ being conjugate to $r>2$. We further introduce $\bar{p} \in V$ as the unique solution of

$$
(\nabla \bar{p}, \nabla v)_{0, \Omega}+(c \bar{p}, v)_{0, \Omega}=\left(y(u)-y^{d}, v\right)_{0, \Omega}, v \in V .
$$

Setting $p:=\bar{p}+\bar{\sigma}$, we have $p \in V_{s}:=W_{0}^{1, s}(\Omega)$. Then, (2.12) gives (2.9), whereas (2.13) and (2.14) imply (2.8). Finally, $\sigma \in \partial I_{K}(u)$ is equivalent to $\langle\sigma, v-y\rangle \leq 0, v \in K$ which proves $\sigma \in \mathcal{M}_{+}(\Omega)$ and (2.10).

We define the active control set $\mathcal{A}(y)$ as the maximal open set $A \subset \Omega$ such that $y(x)=\psi(x)$ f.a.a. $x \in A$ and the inactive control set $\mathcal{I}(y)$ according to $\mathcal{I}(y):=$ $\bigcup_{\varepsilon>0} B_{\varepsilon}$, where $B_{\varepsilon}$ is the maximal open set $B \subset \Omega$ such that $y(x) \leq \psi(x)-\varepsilon$ for almost all $x \in B$.

3. Finite element approximation. We assume that $\left\{\mathcal{T}_{\ell}(\Omega)\right\}$ is a family of shape-regular simplicial triangulations of $\Omega$ which align with $\Gamma_{D}, \Gamma_{N}$ on $\Gamma$. We refer to $\mathcal{N}_{\ell}(D)$ and $\mathcal{E}_{\ell}(D), D \subseteq \bar{\Omega}$, as the sets of vertices and edges of $\mathcal{T}_{\ell}(\Omega)$ in $D \subseteq \bar{\Omega}$. We denote by $h_{T}$ and $|T|$ the diameter and area of an element $T \in \mathcal{T}_{\ell}(\Omega)$ and by $h_{E}$ the length of an edge $E \in \mathcal{E}_{\ell}(D)$. For $E \in \mathcal{E}_{\ell}(\Omega)$ such that $E=T_{+} \cap T_{-}, T_{ \pm} \in \mathcal{T}_{\ell}(\Omega)$, we define $\omega_{E}:=T_{+} \cup T_{-}$as the associated patch.

Throughout the paper, we will also use the following notation: If $A$ and $B$ are two quantities, we say $A \preceq B$, if there exists a positive constant $C$ that only depends 
on the shape regularity of the triangulations but not on their granularities such that $A \leq C B$.

The state constrained optimal control problem (2.2)-(2.4) is discretized by continuous piecewise linear finite elements with respect to the triangulation $\mathcal{T}_{\ell}(\Omega)$. In particular, we refer to $S_{\ell}:=\left\{v_{\ell} \in C_{0}(\Omega)\left|v_{\ell}\right|_{T} \in P_{1}(T), T \in \mathcal{T}_{\ell}(\Omega)\right\}$ as the finite element space spanned by the canonical nodal basis functions $\varphi_{\ell}^{p}, p \in \mathcal{N}_{\ell}(\Omega)$, associated with the nodal points in $\bar{\Omega}$ and to $V_{\ell}$ as its subspace $V_{\ell}:=\left\{v_{\ell} \in S_{\ell}\left|v_{\ell}\right|_{\Gamma_{D}}=0\right\}$. Moreover, we denote by $W_{\ell}:=\left\{w_{\ell} \in L^{2}(\Omega)\left|w_{\ell}\right|_{T} \in P_{0}(T), T \in \mathcal{T}_{\ell}(\Omega)\right\}$ the linear space of elementwise constant functions on $\Omega$.

Given some approximation $u_{\ell}^{d} \in V_{\ell}$ of $u^{d}$, we refer to $J_{\ell}: V_{\ell} \times S_{\ell} \rightarrow \mathbb{R}$ as the discrete objective functional

$$
J_{\ell}\left(y_{\ell}, u_{\ell}\right):=\frac{1}{2}\left\|y_{\ell}-y^{d}\right\|_{0, \Omega}^{2}+\frac{\alpha}{2}\left\|u_{\ell}-u_{\ell}^{d}\right\|_{0, \Omega}^{2} .
$$

Further, we denote by $\psi_{\ell} \in V_{\ell}$ the $V_{\ell}$-interpoland of $\psi$ which is well defined, since $\psi \in C(\bar{\Omega})$.

The finite element approximation of the state constrained optimal control problem (2.2)-(2.4) reads as follows: Find $\left(y_{\ell}, u_{\ell}\right) \in V_{\ell} \times S_{\ell}$ such that

$$
\inf _{y_{\ell}, u_{\ell}} J_{\ell}\left(y_{\ell}, u_{\ell}\right) \text {, }
$$

subject to the constraints

$$
\begin{aligned}
& \left(\nabla y_{\ell}, \nabla v_{\ell}\right)_{0, \Omega}+\left(c y_{\ell}, v_{\ell}\right)_{0, \Omega}=\left(u_{\ell}, v_{\ell}\right)_{0, \Omega} \quad, \quad v_{\ell} \in V_{\ell}, \\
& y_{\ell} \in K_{\ell}:=\left\{v_{\ell} \in V_{\ell} \mid v_{\ell} \leq \psi_{\ell}\right\} .
\end{aligned}
$$

As in the continuous setting, the discrete state constrained optimal control problem (3.2)-(3.4) admits a unique solution $\left(y_{\ell}, u_{\ell}\right) \in K_{\ell} \times S_{\ell}$.

We further choose $\mathcal{M}_{\ell} \subset \mathcal{M}(\Omega)$ according to

$$
\mathcal{M}_{\ell}:=\left\{\mu_{\ell} \in \mathcal{M}(\Omega) \mid \mu_{\ell}=\sum_{p \in \mathcal{N}_{\ell}\left(\Omega \cup \Gamma_{N}\right)} \kappa_{p} \delta_{p}, \kappa_{p} \in \mathbb{R}\right\},
$$

where $\delta_{p}$ stands for the Dirac delta function associated with the nodal point $p$. We obtain the discrete optimality conditions:

Theorem 3.1. Let $\left(y_{\ell}, u_{\ell}\right) \in K_{\ell} \times S_{\ell}$ be the unique solution of (3.2)-(3.4). Then, there exist a discrete adjoint state $p_{\ell} \in V_{\ell}$ and a discrete multiplier $\sigma_{\ell} \in \mathcal{M}_{\ell} \cap \mathcal{M}_{+}(\Omega)$ such that

$$
\begin{aligned}
\left(\nabla y_{\ell}, \nabla v_{\ell}\right)_{0, \Omega}+\left(c y_{\ell}, v_{\ell}\right)_{0, \Omega} & =\left(u_{\ell}, v_{\ell}\right)_{0, \Omega}, v_{\ell} \in V_{\ell}, \\
\left(\nabla p_{\ell}, \nabla v_{\ell}\right)_{0, \Omega}+\left(c p_{\ell}, v_{\ell}\right)_{0, \Omega} & =\left(y_{\ell}-y^{d}, v_{\ell}\right)_{0, \Omega}+\left\langle\sigma_{\ell}, v_{\ell}\right\rangle, v_{\ell} \in V_{\ell}, \\
p_{\ell}+\alpha\left(u_{\ell}-u_{\ell}^{d}\right) & =0 \\
\left\langle\sigma_{\ell}, y_{\ell}-\psi_{\ell}\right\rangle & =0 .
\end{aligned}
$$

Proof. For a proof we refer to [10].

As in the continuous regime, we introduce a regularized discrete multiplier $\bar{\sigma}_{\ell} \in V_{\ell}$ as the solution of

$$
\left(\nabla \bar{\sigma}_{\ell}, v_{\ell}\right)_{0, \Omega}+\left(c \bar{\sigma}_{\ell}, v_{\ell}\right)_{0, \Omega}=\left\langle\sigma_{\ell}, v_{\ell}\right\rangle, v_{\ell} \in V_{\ell}
$$


and define $\bar{p}_{\ell}:=p_{\ell}-\bar{\sigma}_{\ell}$ so that $\bar{p}_{\ell} \in V_{\ell}$ satisfies the discrete analogue of (2.14), i.e.,

$$
\left(\nabla \bar{p}_{\ell}, v_{\ell}\right)_{0, \Omega}+\left(c \bar{p}_{\ell}, v_{\ell}\right)_{0, \Omega}=\left(y_{\ell}-y^{d}, v_{\ell}\right)_{0, \Omega}, v_{\ell} \in V_{\ell}
$$

We further define $\mathcal{A}\left(y_{\ell}\right)$ and $\mathcal{I}\left(y_{\ell}\right)$ as the discrete active and inactive control sets according to

$$
\begin{aligned}
& \mathcal{A}\left(y_{\ell}\right):=\bigcup\left\{T \in \mathcal{T}_{\ell}(\Omega) \mid y_{\ell}(p)=\psi_{\ell}(p) \text { for all vertices } p \in \mathcal{N}_{\ell}(T)\right\}, \\
& \mathcal{I}\left(y_{\ell}\right):=\bigcup\left\{T \in \mathcal{T}_{\ell}(\Omega) \mid y_{\ell}(p)<\psi_{\ell}(p) \text { for at least one vertex } p \in \mathcal{N}_{\ell}(T)\right\} .
\end{aligned}
$$

4. Residual-type a posteriori error estimator. We introduce a residualtype a posteriori error estimator

$$
\eta_{\ell}:=\eta_{\ell}(y)+\eta_{\ell}(\bar{p})
$$

in terms of estimators $\eta_{\ell}(y)$ and $\eta_{\ell}(\bar{p})$ for the state $y$ and the modified adjoint state $\bar{p}$ which consist of element and edge residuals according to

$$
\begin{aligned}
& \eta_{\ell}(y):=\left(\sum_{T \in \mathcal{T}_{\ell}(\Omega)} \eta_{T}^{2}(y)+\sum_{E \in \mathcal{E}_{\ell}(\Omega)} \eta_{E}^{2}(y)\right)^{1 / 2}, \\
& \eta_{\ell}(\bar{p}):=\left(\sum_{T \in \mathcal{T}_{\ell}(\Omega)} \eta_{T}^{2}(\bar{p})+\sum_{E \in \mathcal{E}_{\ell}(\Omega)} \eta_{E}^{2}(\bar{p})\right)^{1 / 2} .
\end{aligned}
$$

The element residuals $\eta_{T}(y)$ and $\eta_{T}(\bar{p}), T \in \mathcal{T}_{\ell}(\Omega)$, are weighted elementwise $L^{2}$ residuals with respect to the strong form of the state equation (2.3) and the modified adjoint state equation (2.14), respectively:

$$
\eta_{T}(y):=h_{T}\left\|c y_{\ell}-u_{\ell}\right\|_{0, T}, \eta_{T}(\bar{p}):=h_{T}\left\|c \bar{p}_{\ell}-\left(y_{\ell}-y^{d}\right)\right\|_{0, T}, T \in \mathcal{T}_{\ell}(\Omega)
$$

The edge residuals $\eta_{E}(y)$ and $\eta_{E}(\bar{p}), E \in \mathcal{E}_{\ell}(\Omega)$, are weighted $L^{2}$-norms of the jumps $\nu_{E} \cdot\left[\nabla y_{\ell}\right]$ and $\nu_{E} \cdot\left[\nabla \bar{p}_{\ell}\right]$ of the normal derivatives across the interior edges

$$
\eta_{E}(y):=h_{E}^{1 / 2}\left\|\nu_{E} \cdot\left[\nabla y_{\ell}\right]\right\|_{0, E}, \eta_{E}(\bar{p}):=h_{E}^{1 / 2}\left\|\nu_{E} \cdot\left[\nabla \bar{p}_{\ell}\right]\right\|_{0, E} .
$$

Denoting by $y_{\ell}^{d} \in W_{\ell}$ some approximation of the desired state $y^{d}$, we further have to take into account data oscillations with respect to the data $u^{d}, y^{d}$ of the problem

$$
o s c_{\ell}:=\left(o s c_{\ell}^{2}\left(u^{d}\right)+o s c_{\ell}^{2}\left(y^{d}\right)\right)^{1 / 2},
$$

where $0 s c_{\ell}\left(u^{d}\right)$ and $o s c_{\ell}\left(y^{d}\right)$ are given by

$$
\begin{aligned}
& \operatorname{osc}_{\ell}\left(u^{d}\right):=\left(\sum_{T \in \mathcal{T}_{\ell}(\Omega)} \operatorname{osc}_{T}^{2}\left(u^{d}\right)\right)^{1 / 2}, \operatorname{osc}_{T}\left(u^{d}\right):=\left\|u^{d}-u_{\ell}^{d}\right\|_{0, T}, T \in \mathcal{T}_{\ell}(\Omega) \\
& o s c_{\ell}\left(y^{d}\right):=\left(\sum_{T \in \mathcal{T}_{\ell}(\Omega)} \operatorname{osc}_{T}^{2}\left(y^{d}\right)\right)^{1 / 2}, \operatorname{osc}_{T}\left(y^{d}\right):=h_{T}\left\|y^{d}-y_{\ell}^{d}\right\|_{0, T}, T \in \mathcal{T}_{\ell}(\Omega) .
\end{aligned}
$$

For standard finite element discretizations of second order elliptic boundary value problems, it can be shown that residual-type a posteriori error estimators such as (4.2),(4.3) provide and upper and a lower bound for the global discretization error up 
to data oscillations. In this paper, we want to establish a similar result for the global discretization errors in the state, the adjoint state, and the control. To this end, we introduce

$$
e_{y}:=y-y_{\ell} \quad, \quad e_{p}:=p-p_{\ell} \quad, \quad e_{u}:=u-u_{\ell} .
$$

As in the case of finite element discretizations of control constrained elliptic boundary value problems (cf. $[18,22]$ ), the a posteriori error analysis involves an auxiliary state $y\left(u_{\ell}\right) \in V$ and an auxiliary adjoint state $\bar{p}\left(y_{\ell}\right) \in V$ which are defined according to

$$
\begin{aligned}
\left(\nabla y\left(u_{\ell}\right), \nabla v\right)_{0, \Omega}+\left(c y\left(u_{\ell}\right), v\right)_{0, \Omega} & =\left(u_{\ell}, v\right)_{0, \Omega}, v \in V, \\
\left(\nabla \bar{p}\left(y_{\ell}\right), \nabla v\right)_{0, \Omega}+\left(c \bar{p}\left(y_{\ell}\right), v\right)_{0, \Omega} & =\left(y_{\ell}-y^{d}, v\right)_{0, \Omega}, v \in V .
\end{aligned}
$$

We note that $y\left(u_{\ell}\right), \bar{p}\left(y_{\ell}\right) \in V \cap W^{1, r}(\Omega)$ due the assumption on the regularity of the solutions of the associated elliptic boundary value problems. We also introduce an auxiliary discrete state $y_{\ell}(u) \in V_{\ell}$ as the solution of the finite dimensional variational problem

$$
\left(\nabla y_{\ell}(u), \nabla v_{\ell}\right)_{0, \Omega}+\left(c y_{\ell}(u), v_{\ell}\right)_{0, \Omega}=\left(u, v_{\ell}\right)_{0, \Omega}, v_{\ell} \in V_{\ell} .
$$

The auxiliary states $y\left(u_{\ell}\right) \in V$ and $y_{\ell}(u) \in V_{\ell}$ do not necessarily satisfy the state constraints, i.e., it may happen that $y\left(u_{\ell}\right) \notin K$ or $y_{\ell}(u) \notin K_{\ell}$. Therefore, we introduce the consistency error

$$
e_{c}\left(u, u_{\ell}\right):=\max \left(\left\langle\sigma_{\ell}, y_{\ell}(u)-\psi\right\rangle+\left\langle\sigma, y\left(u_{\ell}\right)-\psi\right\rangle, 0\right) .
$$

We note that for $u=u_{\ell}$ we have $e_{c}\left(u, u_{\ell}\right)=0$, since in this case $y\left(u_{\ell}\right)=y$ and $y_{\ell}(u)=y_{\ell}$, and hence, $e_{c}\left(u, u_{\ell}\right)=0$ due to (2.10) and (3.9). We thus define

$$
\tilde{e}_{c}\left(u, u_{\ell}\right):=\left\{\begin{array}{r}
e_{c}\left(u, u_{\ell}\right) /\left\|u-u_{\ell}\right\|_{0, \Omega}, u \neq u_{\ell} \\
0, u=u_{\ell}
\end{array} .\right.
$$

The refinement of a triangulation $\mathcal{T}_{\ell}(\Omega)$ is based on bulk criteria that have been previously used in the convergence analysis of adaptive finite element for nodal finite element methods [11, 29]. For the state-constrained optimal control problem under consideration, the bulk criteria are as follows: Given universal constants $\Theta_{i} \in$ $(0,1), 1 \leq i \leq 4$, we create a set of edges $\mathcal{M}^{E} \subset \mathcal{E}_{h}(\Omega)$ and sets of elements $\mathcal{M}^{\eta, T}, \mathcal{M}^{o s c_{1}, T}, \mathcal{M}^{o s c_{2}, T} \subset \mathcal{T}_{\ell}(\Omega)$ such that

$$
\begin{aligned}
\Theta_{1} \sum_{E \in \mathcal{E}_{\ell}(\Omega)}\left(\eta_{y, E}^{2}+\eta_{\bar{p}, E}^{2}\right) & \leq \sum_{E \in \mathcal{M}^{E}}\left(\eta_{y, E}^{2}+\eta_{\bar{p}, E}^{2}\right), \\
\Theta_{2} \sum_{T \in \mathcal{T}_{\ell}(\Omega)}\left(\eta_{y, T}^{2}+\eta_{\bar{p}, T}^{2}\right) & \leq \sum_{T \in \mathcal{M}^{\eta, T}}\left(\eta_{y, T}^{2}+\eta_{\bar{p}, T}^{2}\right), \\
\Theta_{3} \sum_{T \in \mathcal{T}_{\ell}(\Omega)} o s c_{T}^{2}\left(u^{d}\right) & \leq \sum_{T \in \mathcal{M}^{o s c_{1}, T}} o s c_{T}^{2}\left(u^{d}\right), \\
\Theta_{4} \sum_{T \in \mathcal{T}_{\ell}(\Omega)} o s c_{T}^{2}\left(y^{d}\right) & \leq \sum_{T \in \mathcal{M}^{o s c_{2}, T}} o s c_{T}^{2}\left(y^{d}\right) .
\end{aligned}
$$

The bulk criteria are realized by a greedy algorithm (cf., e.g., [18]). We set

$$
\mathcal{M}^{T}:=\mathcal{M}^{\eta, T} \cup \mathcal{M}^{o s c_{1}, T} \cup \mathcal{M}^{o s c_{2}, T}
$$


and refine an element $T \in \mathcal{T}_{\ell}(\Omega)$ by bisection (i.e., by joining the midpoint of the longest edge with the opposite vertex), if $T \in \mathcal{M}^{T}$ and an edge $E \in \mathcal{E}_{\ell}(T)$ by bisection (joining its midpoint with the opposite vertices of the adjacent elements), if $E \in \mathcal{M}^{E}$. Denoting by $\mathcal{N}_{T}:=\left\{T^{\prime} \in \mathcal{T}_{\ell}(\Omega) \mid T^{\prime} \cap T \neq \emptyset\right\}$ the set of all neighboring triangles of $T \in \mathcal{T}_{\ell}(\Omega)$, we define the set

$$
\mathcal{F}_{\ell}\left(y_{\ell}\right):=\partial \mathcal{A}\left(y_{\ell}\right) \cup \partial \mathcal{I}\left(y_{\ell}\right)
$$

where

$$
\begin{aligned}
\partial \mathcal{A}\left(y_{\ell}\right) & :=\bigcup\left\{T \subset \mathcal{A}\left(y_{\ell}\right) \mid \mathcal{N}_{T} \cap \mathcal{I}\left(y_{\ell}\right) \neq \emptyset\right\} \\
\partial \mathcal{I}\left(y_{\ell}\right) & :=\bigcup\left\{T \subset \mathcal{I}\left(y_{\ell}\right) \mid \mathcal{N}_{T} \cap \mathcal{A}\left(y_{\ell}\right) \neq \emptyset\right\} .
\end{aligned}
$$

The set $\mathcal{F}_{\ell}\left(y_{\ell}\right)$ represents a neighborhood of the discrete free boundary between the discrete active and inactive sets $\mathcal{A}\left(y_{\ell}\right)$ and $\mathcal{I}\left(y_{\ell}\right)$. In order to guarantee a sufficient resolution of the continuous free boundary between $\mathcal{A}(y)$ and $\mathcal{I}(y)$, at each refinement step, the elements $T \in \mathcal{F}_{\ell}\left(u_{\ell}\right)$ are refined by bisection.

5. Reliability of the estimator. We prove reliability of the residual-type error estimator (4.1) in the sense that it provides an upper bound for the discretization errors $e_{y}, e_{u}$, and $e_{\bar{p}}:=\bar{p}-\bar{p}_{\ell}$ up to the data oscillations $\operatorname{osc}_{\ell}\left(u^{d}\right)$ and $\operatorname{osc}_{\ell}\left(y^{d}\right)$ and the consistency error $\tilde{e}_{c}\left(u, u_{\ell}\right)$. Since the adjoint state $p$ and the discrete adjoint state $p_{\ell}$ are related to the control $u$ and the discrete control $u_{\ell}$ by means of the fundamental relationships (2.9) and (3.8), this leads to an upper bound for the $L^{2}$-norm of the discretization error $e_{p}$ as well.

THEOREM 5.1. Let $(y, u, p, \sigma)$ and $\left(y_{\ell}, u_{\ell}, p_{\ell}, \sigma_{\ell}\right)$ be the solutions of (2.7)-(2.10) and (3.6)-(3.9) and let $\eta_{\ell}$, osc $_{\ell}\left(u^{d}\right)$, and $\tilde{e}_{c}\left(u, u_{\ell}\right)$ be the error estimator, the data oscillation, and the consistency error according to (4.1),(4.7) and (4.14), respectively. Further, let $\bar{p}$ and $\bar{p}_{\ell}$ be the modified adjoint states as given by (2.14),(3.11). Then, there holds

$$
\left\|e_{y}\right\|_{1, \Omega}+\left\|e_{u}\right\|_{0, \Omega}+\left\|e_{\bar{p}}\right\|_{1, \Omega}+\left\|e_{p}\right\|_{0, \Omega} \preceq \eta+\operatorname{osc}_{\ell}\left(u^{d}\right)+\tilde{e}_{c}\left(u, u_{\ell}\right) .
$$

The proof of Theorem 5.1 will be given by the following two Lemmas 5.2 and 5.3.

LEMMA 5.2. In addition to the assumptions of Theorem 5.1 let $y\left(u_{\ell}\right)$ and $\bar{p}\left(y_{\ell}\right)$ be the auxiliary state and auxiliary adjoint state according to (4.10),(4.11). Then, there holds

$$
\begin{aligned}
& \left\|e_{y}\right\|_{1, \Omega}+\left\|e_{\bar{p}}\right\|_{1, \Omega}+\left\|e_{u}\right\|_{0, \Omega} \preceq \\
& \preceq\left\|y\left(u_{\ell}\right)-y_{\ell}\right\|_{1, \Omega}+\left\|\bar{p}\left(y_{\ell}\right)-\bar{p}_{\ell}\right\|_{1, \Omega}+\operatorname{osc}_{\ell}\left(u^{d}\right)+\tilde{e}_{c}\left(u, u_{\ell}\right) .
\end{aligned}
$$

Proof. Obviously, $e_{y}$ and $e_{\bar{p}}$ can be estimated from above by

$$
\begin{aligned}
&\left\|e_{y}\right\|_{1, \Omega} \leq\left\|y-y\left(u_{\ell}\right)\right\|_{1, \Omega}+\left\|y\left(u_{\ell}\right)-y_{\ell}\right\|_{1, \Omega}, \\
&\left\|e_{\bar{p}}\right\|_{1, \Omega} \leq\left\|\bar{p}-\bar{p}\left(y_{\ell}\right)\right\|_{1, \Omega}+\left\|\bar{p}\left(y_{\ell}\right)-\bar{p}_{\ell}\right\|_{1, \Omega} .
\end{aligned}
$$

Setting $v=y-y\left(u_{\ell}\right)$ in (2.7),(4.10), and $M:=C / \gamma$ with $\gamma, C$ from (2.1), for the first term on the right-hand side in (5.3) we readily get

$$
\left\|y-y\left(u_{\ell}\right)\right\|_{1, \Omega}^{2} \leq M\left\|e_{u}\right\|_{0, \Omega}\left\|y-y\left(u_{\ell}\right)\right\|_{0, \Omega} \leq M\left\|e_{u}\right\|_{0, \Omega}\left\|y-y\left(u_{\ell}\right)\right\|_{1, \Omega},
$$


and hence,

$$
\left\|y-y\left(u_{\ell}\right)\right\|_{1, \Omega} \leq M\left\|e_{u}\right\|_{0, \Omega} .
$$

Likewise, choosing $v=\bar{p}-\bar{p}\left(y_{\ell}\right)$ in (2.14) and (4.11), for the first term on the righthand side in (5.4) it follows that

$$
\left\|\bar{p}-\bar{p}\left(y_{\ell}\right)\right\|_{1, \Omega}^{2} \leq M\left\|e_{y}\right\|_{0, \Omega}\left\|\bar{p}-\bar{p}\left(y_{\ell}\right)\right\|_{0, \Omega} \leq M\left\|e_{y}\right\|_{1, \Omega}\left\|\bar{p}-\bar{p}\left(y_{\ell}\right)\right\|_{1, \Omega} .
$$

Consequently, in view of (5.3) and (5.5) we obtain

$$
\left\|\bar{p}-\bar{p}\left(y_{\ell}\right)\right\|_{1, \Omega} \leq M\left\|e_{y}\right\|_{1, \Omega} \leq M^{2}\left\|e_{u}\right\|_{0, \Omega}+M\left\|y\left(u_{\ell}\right)-y_{\ell}\right\|_{1, \Omega} .
$$

It remains to estimate $\left\|e_{u}\right\|_{0, \Omega}$. Taking advantage of (2.9) and (3.8) and observing $p=\bar{p}+\bar{\sigma}, p_{\ell}=\bar{p}_{\ell}+\bar{\sigma}_{\ell}$, we find

$$
\begin{aligned}
\left\|e_{u}\right\|_{0, \Omega}^{2} & =\left(e_{u}, u^{d}-u_{\ell}^{d}\right)_{0, \Omega}-\frac{1}{\alpha}\left(e_{u}, e_{p}\right)_{0, \Omega}= \\
& =\left(e_{u}, u^{d}-u_{\ell}^{d}\right)_{0, \Omega}+\frac{1}{\alpha}\left(\left(e_{u}, \bar{p}_{\ell}-\bar{p}\right)_{0, \Omega}+\left(e_{u}, \bar{\sigma}_{\ell}-\bar{\sigma}\right)_{0, \Omega}\right) .
\end{aligned}
$$

Using Young's inequality, the first term on the right-hand side in (5.7) can be easily estimated according to

$$
\begin{aligned}
\left(e_{u}, u^{d}-u_{\ell}^{d}\right)_{0, \Omega} & =\sum_{T \in \mathcal{T}_{\ell}(\Omega)}\left(e_{u}, u^{d}-u_{\ell}^{d}\right)_{0, T} \leq \\
& \leq\left(\sum_{T \in \mathcal{T}_{\ell}(\Omega)}\left\|e_{u}\right\|_{0, T}^{2}\right)^{1 / 2}\left(\sum_{T \in \mathcal{T}_{\ell}(\Omega)} \operatorname{osc}_{T}\left(u^{d}\right)^{2}\right)^{1 / 2}= \\
& =\left\|e_{u}\right\|_{0, \Omega} \operatorname{osc}_{\ell}\left(u^{d}\right) \leq \frac{1}{8}\left\|e_{u}\right\|_{0, \Omega}^{2}+2 \operatorname{osc}_{\ell}^{2}\left(u^{d}\right) .
\end{aligned}
$$

The second term on the right-hand side in (5.7) will be split by means of

$$
\left(e_{u}, \bar{p}_{\ell}-\bar{p}\right)_{0, \Omega}=\left(e_{u}, \bar{p}_{\ell}-\bar{p}\left(y_{\ell}\right)\right)_{0, \Omega}+\left(e_{u}, \bar{p}\left(y_{\ell}\right)-\bar{p}\right)_{0, \Omega} .
$$

and the resulting two terms will be further estimated separately. Using Young's inequality once more, for the first term we get

$$
\left(e_{u}, \bar{p}_{\ell}-\bar{p}\left(y_{\ell}\right)\right)_{0, \Omega} \leq \frac{\alpha}{8}\left\|e_{u}\right\|_{0, \Omega}^{2}+\frac{2}{\alpha}\left\|\bar{p}_{\ell}-\bar{p}\left(y_{\ell}\right)\right\|_{1, \Omega}^{2} .
$$

On the other hand, setting $v=\bar{p}\left(y_{\ell}\right)-\bar{p}$ in $(2.7),(3.6)$ and $v=y\left(u_{\ell}\right)-y$ in $(2.14),(3.11)$, for the second term it follows that

$$
\begin{aligned}
& \left(e_{u}, \bar{p}\left(y_{\ell}\right)-\bar{p}\right)_{0, \Omega}=\left(y-y_{\ell}, y\left(u_{\ell}\right)-y\right)_{0, \Omega}=-\left\|y-y\left(u_{\ell}\right)\right\|_{0, \Omega}^{2}+ \\
& +\left(y\left(u_{\ell}\right)-y_{\ell}, y\left(u_{\ell}\right)-y\right)_{0, \Omega} \leq\left\|y\left(u_{\ell}\right)-y_{\ell}\right\|_{1, \Omega}\left\|y-y\left(u_{\ell}\right)\right\|_{1, \Omega} \leq \\
& \leq M\left\|y\left(u_{\ell}\right)-y_{\ell}\right\|_{1, \Omega}\left\|e_{u}\right\|_{0, \Omega} \leq \frac{\alpha}{8}\left\|e_{u}\right\|_{0, \Omega}^{2}+\frac{2 M^{2}}{\alpha}\left\|y\left(u_{\ell}\right)-y_{\ell}\right\|_{1, \Omega}^{2},
\end{aligned}
$$

where we further used (5.5) and Young's inequality.

Finally, as far as the third term on the right-hand side in (5.7) is concerned, in view 
of $(2.7),(3.6),(4.10),(4.12)$ as well as $(2.13),(3.10)$ and the complementarity relations (2.10) and (3.9) we obtain

$$
\begin{aligned}
& \left(e_{u}, \bar{\sigma}_{\ell}-\bar{\sigma}\right)_{0, \Omega}= \\
& =\left(\nabla\left(y_{\ell}(u)-y_{\ell}\right), \nabla \bar{\sigma}_{\ell}\right)_{0, \Omega}+\left(c\left(y_{\ell}(u)-y_{\ell}\right), \bar{\sigma}_{\ell}\right)_{0, \Omega}- \\
& -\left(\nabla\left(y-y\left(u_{\ell}\right)\right), \nabla \bar{\sigma}\right)_{0, \Omega}-\left(c\left(y-y\left(u_{\ell}\right)\right), \bar{\sigma}\right)_{0, \Omega}= \\
& =\left\langle\sigma_{\ell}, y_{\ell}(u)-y_{\ell}\right\rangle+\left\langle\sigma, y\left(u_{\ell}\right)-y\right\rangle=\left\langle\sigma_{\ell}, y_{\ell}(u)-\psi\right\rangle+ \\
& +\underbrace{\left\langle\sigma_{\ell}, \psi-y_{\ell}\right\rangle}_{=0}+\left\langle\sigma, y\left(u_{\ell}\right)-\psi\right\rangle+\underbrace{\langle\sigma, \psi-y\rangle}_{=0} \leq \\
& \leq\left\|e_{u}\right\|_{0, \Omega} \tilde{e}_{c}\left(u, u_{\ell}\right) \leq \frac{\alpha}{8}\left\|e_{u}\right\|_{0, \Omega}^{2}+\frac{2}{\alpha} \tilde{e}_{c}^{2}\left(u, u_{\ell}\right) .
\end{aligned}
$$

Using (5.8),(5.10)-(5.12) in (5.7) results in

$$
\left\|e_{u}\right\|_{0, \Omega} \preceq\left\|y\left(u_{\ell}\right)-y_{\ell}\right\|_{1, \Omega}+\left\|\bar{p}\left(y_{\ell}\right)-\bar{p}_{\ell}\right\|_{1, \Omega}+o s c_{\ell}\left(u^{d}\right)+\tilde{e}_{c}\left(u, u_{\ell}\right) .
$$

Collecting the estimates (5.5),(5.6) and (5.13) gives the assertion.

LEMMA 5.3. Under the same assumptions as in Lemma 5.2 there holds

$$
\begin{aligned}
\left\|y\left(u_{\ell}\right)-y_{\ell}\right\|_{1, \Omega} \preceq \eta_{\ell}(y), \\
\left\|\bar{p}\left(y_{\ell}\right)-\bar{p}_{\ell}\right\|_{1, \Omega} \preceq \eta_{\ell}(\bar{p}) .
\end{aligned}
$$

Proof. Due to Galerkin orthogonality, the assertion follows by standard arguments from the a posteriori error analysis of adaptive finite element methods (see, e.g., [32]).

Proof of Theorem 5.1. Combining the results from Lemma 5.2 and Lemma 5.3, we obtain

$$
\left\|e_{y}\right\|_{1, \Omega}+\left\|e_{u}\right\|_{0, \Omega}+\left\|e_{\bar{p}}\right\|_{1, \Omega} \preceq \eta_{\ell}+\operatorname{osc}_{\ell}\left(u^{d}\right)+\tilde{e}_{c}\left(u, u_{\ell}\right) .
$$

In particular, this estimate is satisfied by each norm on the left-hand side. In view of $(2.9),(3.8)$ we have $e_{p}=\alpha\left(u_{\ell}-u\right)+\alpha\left(u^{d}-u_{\ell}^{d}\right)$ whence

$$
\left\|e_{p}\right\|_{0, \Omega} \preceq\left\|e_{u}\right\|_{0, \Omega}+\operatorname{osc}_{\ell}\left(u^{d}\right) .
$$

Then, (5.1) is a direct consequence of (5.16) and (5.17).

6. Efficiency of the estimator. In this section, we show that up to data oscillations the error estimator $\eta$ also provides a lower bound for the discretization errors in the state, the modified adjoint state and the control.

THeOREM 6.1. Let $(y, u, p, \sigma)$ and $\left(y_{\ell}, u_{\ell}, p_{\ell}, \sigma_{\ell}\right)$ be the solutions of (2.7)-(2.10) and (3.6)-(3.9) and let $\eta_{\ell}$ and $\operatorname{osc}_{\ell}\left(y^{d}\right)$ be the error estimator and the data oscillation as given by (4.1) and (4.7), respectively. Further, let $\bar{p}$ and $\bar{p}_{\ell}$ be the modified adjoint states as given by $(2.14),(3.11)$. Then, there holds

$$
\eta-\operatorname{osc}_{\ell}\left(y^{d}\right) \preceq\left\|e_{y}\right\|_{1, \Omega}+\left\|e_{u}\right\|_{0, \Omega}+\left\|e_{\bar{p}}\right\|_{1, \Omega} .
$$


The proof of Theorem 6.1 will be a direct consequence of the subsequent Lemmas 6.2 and 6.3. In particular, these Lemmas will establish local efficiency of the estimator in the sense that the element and edge residuals can be bounded from above by norms of the discretization errors on the elements and associated patches, respectively.

Lemma 6.2. Let $\eta_{T}(y)$ and $\eta_{T}(\bar{p}), T \in \mathcal{T}_{\ell}(\Omega)$, be the element residuals as given by (4.4). Then, there holds

$$
\begin{aligned}
& \eta_{T}^{2}(y) \preceq\left\|e_{y}\right\|_{1, T}^{2}+h_{T}^{2}\left\|e_{u}\right\|_{0, T}^{2}, \\
& \eta_{T}^{2}(\bar{p}) \preceq\left\|e_{\bar{p}}\right\|_{1, T}^{2}+h_{T}^{2}\left\|e_{y}\right\|_{0, T}^{2}+o s c_{T}^{2}\left(y^{d}\right) .
\end{aligned}
$$

Proof. We denote by $\varphi_{T}, T \in \mathcal{T}_{\ell}(\Omega)$, the element bubble function given by the product of the barycentric coordinates associated with the vertices of $T$ and set $z_{\ell}:=$ $\left(u_{\ell}-c y_{\ell}\right) \varphi_{T}$. Taking advantage of the fact that $z_{\ell}$ is an admissible test function in $(2.7)$ and $\left.\Delta y_{\ell}\right|_{T}=0$, we obtain

$$
\begin{aligned}
\eta_{T}^{2}(y) & \preceq h_{T}^{2}\left(u_{\ell}-c y_{\ell}, z_{\ell}\right)_{0, T}=h_{T}^{2}\left(\left(u, z_{\ell}\right)_{0, T}+\left(\Delta y_{\ell}-c y_{\ell}, z_{\ell}\right)_{0, T}+\left(u_{\ell}-u, z_{\ell}\right)_{0, T}\right) \\
& =h_{T}^{2}\left(\left(\nabla\left(y-y_{\ell}\right), \nabla z_{\ell}\right)_{0, T}+\left(c\left(y-y_{\ell}, z_{\ell}\right)_{0, T}+\left(u_{\ell}-u, z_{\ell}\right)_{0, T}\right) .\right.
\end{aligned}
$$

Using standard estimates for $\left\|\nabla z_{\ell}\right\|_{0, T}$ and $\left\|z_{\ell}\right\|_{0, T}$ (cf., e.g., [32]) readily gives (6.2). The proof of (6.3) follows along the same lines.

Lemma 6.3 . Let $\eta_{T}(y), \eta_{T}(\bar{p}), T \in \mathcal{T}_{\ell}(\Omega)$, and $\eta_{E}(y), \eta_{E}(\bar{p}), E \in \mathcal{E}_{\ell}(\Omega)$, be the element and edge residuals as given by (4.4),(4.5). Further, let $\operatorname{osc}_{T}\left(y^{d}\right), T \in \mathcal{T}_{\ell}(\Omega)$, be the element contribution to the data oscillation in $y^{d}$ according to (4.8). Then, there holds

$$
\begin{aligned}
& \eta_{E}^{2}(y) \preceq\left\|e_{y}\right\|_{1, \omega_{E}}^{2}+h_{E}^{2}\left\|e_{u}\right\|_{0, \omega_{E}}^{2}+\eta_{\omega_{E}}^{2}(y), \\
& \eta_{E}^{2}(\bar{p}) \preceq\left\|e_{\bar{p}}\right\|_{1, \omega_{E}}^{2}+h_{E}^{2}\left\|e_{y}\right\|_{0, \omega_{E}}^{2}+\eta_{\omega_{E}}^{2}(\bar{p}),
\end{aligned}
$$

where $\eta_{\omega_{E}}(y):=\left(\eta_{T_{+}}^{2}(y)+\eta_{T_{-}}^{2}(y)\right)^{1 / 2}$ and $\eta_{\omega_{E}}(\bar{p})$ are defined analogously.

Proof. We denote by $\varphi_{E}, E \in \mathcal{E}_{\ell}(\Omega)$, the edge bubble function given by the product of the barycentric coordinates associated with the two vertices of $E$. We set $\zeta_{E}:=\left.\left(\nu_{E} \cdot\left[\nabla y_{\ell}\right]\right)\right|_{E}$ and $z_{\ell}:=\tilde{\zeta}_{E} \varphi_{E}$, where is the extension of $\zeta_{E}$ to $\omega_{E}$ as in [32]. Since $z_{\ell}$ is an admissible test function in (2.7) and $\left.\Delta y_{\ell}\right|_{T}=0$, it follows that

$$
\begin{aligned}
\eta_{E}^{2}(y) \preceq h_{E}\left(\nu_{E} \cdot\left[\nabla y_{\ell}\right], \zeta_{E} \varphi_{E}\right)_{0, E}= \\
=h_{E} \sum_{i \in\{+,-\}}\left\{\left(\nu_{\partial T_{i}} \cdot \nabla y_{l}, z_{l}\right)_{0, \partial T_{i}}-\left(\Delta y_{l}, z_{l}\right)_{0, T_{i}}\right\} \\
=h_{E}\left(\left(\nabla\left(y_{\ell}-y\right), \nabla z_{\ell}\right)_{0, \omega_{E}}+\left(c\left(y_{\ell}-y\right), z_{\ell}\right)_{0, \omega_{E}}+\right. \\
\left.\quad\left(u-u_{\ell}, z_{\ell}\right)_{0, \omega_{E}}+\left(u_{\ell}-c y_{\ell}, z_{\ell}\right)_{0, \omega_{E}}\right) .
\end{aligned}
$$

Using standard estimates for $z_{\ell}$ (cf., e.g., [32]) results in (6.4). The estimate (6.5) can be proved in much the same way.

Proof of Theorem 6.1. Summing up the estimates (6.3)-(6.5) over all $T \in \mathcal{T}_{\ell}(\Omega)$ and $E \in \mathcal{E}_{\ell}(\Omega)$, respectively, and using the fact that the union of the patches $\omega_{E}$ has a finite overlap, immediately proves (6.1). 
7. Numerical results. In this section, we illustrate the performance of the residual-type a posteriori error estimator by two representative numerical examples. The first example features a solution that strongly oscillates around the origin.

\section{Example 1: Constant Obstacle}

The data of the problem are as follows

$$
\begin{aligned}
& \Omega:=(-2,2)^{2}, \quad y^{d}:=y(r)+\Delta p(r)+\sigma(r) \quad, \quad u^{d}:=u(r)+\alpha^{-1} p(r), \\
& \psi:=0 \quad, \quad \alpha:=0.1 \quad, \quad c=0 \quad, \quad \Gamma_{D}:=\partial \Omega .
\end{aligned}
$$

Here, $y=y(r), u=u(r), p=p(r)$, and $\sigma=\sigma(r), r:=\left(x_{1}^{2}+x_{2}^{2}\right)^{1 / 2},\left(x_{1}, x_{2}\right)^{T} \in \Omega$, are chosen according to

$$
\begin{array}{rlrl}
y(r):=-r^{\frac{4}{3}} \gamma_{1}(r) & , & u(r):=-\Delta y(r), \\
p(r):=\gamma_{2}(r)\left(r^{4}-\frac{3}{2} r^{3}+\frac{9}{16} r^{2}\right) & , \quad \sigma(r):=\left\{\begin{array}{c}
0, r<0.75 \\
0.1, \text { otherwise }
\end{array},\right.
\end{array}
$$

where

$$
\begin{aligned}
\gamma_{1} & :=\left\{\begin{array}{l}
1, r<0.25 \\
-192(r-0.25)^{5}+240(r-0.25)^{4}-80(r-0.25)^{3}+1,0.25<r<0.75 \\
0, \text { otherwise }
\end{array}\right. \\
\gamma_{2}: & :\left\{\begin{array}{l}
1, r<0.75 \\
0, \text { otherwise }
\end{array} .\right.
\end{aligned}
$$

It is easy to check that the above functions satisfy the optimality conditions (2.7)(2.10).

Figures 7.1, 7.2 and 7.3 display the desired state $y^{d}$, the control shift $u^{d}$, the control $u_{l}$, the state $y_{l}$, the adjoint state $p_{l}$ and the modified adjoint state $\bar{p}_{l}$, respectively, on an adaptively generated net with 11775 nodes.

The initial simplicial triangulation $\mathcal{T}_{h_{0}}$ was chosen according to a subdivision of $\Omega$ by joining the four vertices resulting in four congruent triangles and one interior nodal point. The parameters $\Theta_{i}$ in the bulk criterion have been specified according to $\Theta_{i}=0.7,1 \leq i \leq 4$. Figure 7.4 shows the adaptively generated triangulations after twelve (left) and fourteen (right) refinement steps.
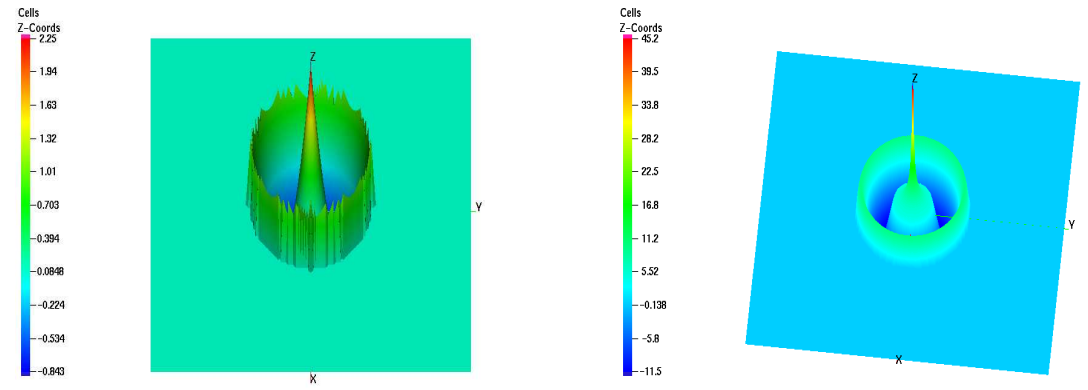

FIG. 7.1. Example 1: Visualization of the desired state $y^{d}$ (left) and the control shift $u^{d}$ (right)) 

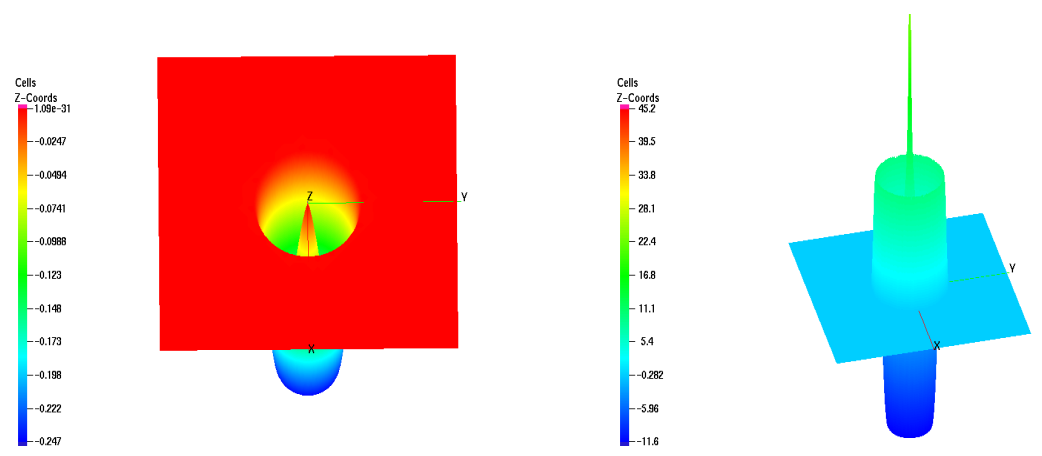

FIG. 7.2. Example 1: Visualization of the discrete state $y_{l}$ (left) and the discrete control $u_{l}$ (right) on a triangulation with 11775 nodes
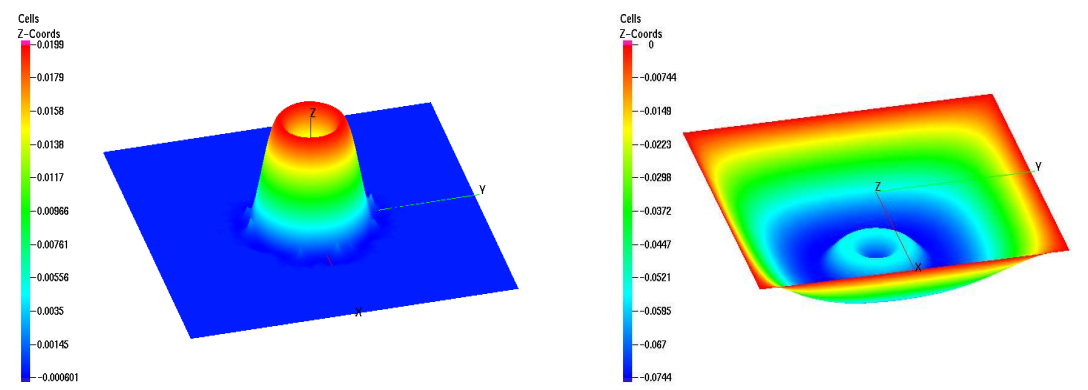

FIG. 7.3. Example 1: Visualization of the discrete adjoint state $p$ (left) and the discrete modified adjoint state $\bar{p}$ (right) on a triangulation with 11775 nodes.

More detailed information is given in Table 1 - Table 4. In particular, Table 1 displays the error reduction in the total error

$$
\left\|z-z_{l}\right\|:=\left|y-y_{l}\right|_{1, \Omega}+\left\|u-u_{l}\right\|_{0, \Omega}
$$

and the $H^{1}$-error in the state, the $L^{2}$-errors in the control, and in the adjoint state, and the $H^{1}$-error in the modified adjoint state, respectively. The actual components $\eta_{y}, \eta_{\bar{p}}$ of the residual type a posteriori error estimator, the data oscillations $\operatorname{osc}_{h}\left(u^{d}\right), \operatorname{osc}_{h}\left(y^{d}\right)$, and the consistency error $\tilde{e}_{c}\left(u, u_{h}\right)$ are given in Table 2, whereas Table 3 contains the average values of the local element and edge contributions of the error estimator as well as the average values of the data oscillations. Finally, Table 4 lists the percentages of elements and edges that have been marked for refinement according to the bulk criteria. Here, $M^{f b, T}, M^{\eta, T}, M^{o s c_{1}, T}$ and $M^{o s c_{2}, T}$ stand for the level $\ell$ elements marked for refinement due to the resolution of the free boundary, the element residuals, and the data oscillations, respectively, whereas $M^{\eta, E}$ refers to the edges marked for refinement with regard to the edge residuals. On the two coarsest grid, the sum of the percentages exceeds $100 \%$, since an element $T \in \mathcal{T}_{h}(\Omega)$ may satisfy more than one criterion in the bulk criteria. We see that at the very beginning the refinement is dominated by the resolution of the free boundary, whereas at later stages the element residuals prevail. The edge residuals do not play a dominant role, since in this example both the state and the modified adjoint state are smooth. 

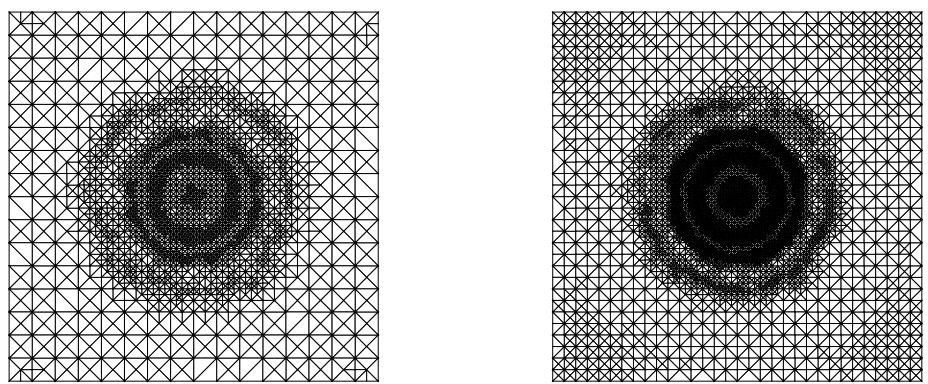

FIG. 7.4. Example 1: Adaptively generated grid after 12 (left) and 14 (right) refinement steps, $\Theta_{i}=0.7$

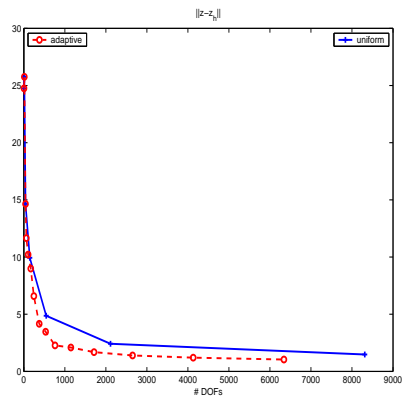

FIG. 7.5. Example 1: Adaptive versus uniform refinement, $\Theta_{i}=0.7$

Figure 7.5 shows the benefit of adaptive versus uniform refinement by displaying the total discretization error as a function of the number of degrees of freedom.

TABLE 7.1

Example 1: Convergence history of the adaptive FEM, Part I: Total discretization error and discretization errors in the state, the control, the adjoint state, and the modified adjoint state

\begin{tabular}{|r|r||c|c|c|c|c|}
\hline$\ell$ & $N_{\text {dof }}$ & $\left\|z-z_{\ell}\right\|$ & $\left|y-y_{\ell}\right|_{1}$ & $\left\|u-u_{\ell}\right\|_{0}$ & $\left\|p-p_{\ell}\right\|_{0}$ & $\left|\bar{p}-\bar{p}_{\ell}\right|_{1}$ \\
\hline 0 & 5 & $2.48 \mathrm{e}+01$ & $2.13 \mathrm{e}+00$ & $2.11 \mathrm{e}+01$ & $9.45 \mathrm{e}-01$ & $7.54 \mathrm{e}-01$ \\
1 & 13 & $2.58 \mathrm{e}+01$ & $1.51 \mathrm{e}+00$ & $2.37 \mathrm{e}+01$ & $2.06 \mathrm{e}+00$ & $6.74 \mathrm{e}-01$ \\
2 & 41 & $1.46 \mathrm{e}+01$ & $1.02 \mathrm{e}+00$ & $1.35 \mathrm{e}+01$ & $1.28 \mathrm{e}-01$ & $1.06 \mathrm{e}-01$ \\
4 & 105 & $1.02 \mathrm{e}+01$ & $7.34 \mathrm{e}-01$ & $9.41 \mathrm{e}+00$ & $9.54 \mathrm{e}-02$ & $7.88 \mathrm{e}-02$ \\
6 & 244 & $6.58 \mathrm{e}+00$ & $5.41 \mathrm{e}-01$ & $6.01 \mathrm{e}+00$ & $4.78 \mathrm{e}-02$ & $6.02 \mathrm{e}-02$ \\
8 & 532 & $3.47 \mathrm{e}+00$ & $2.80 \mathrm{e}-01$ & $3.18 \mathrm{e}+00$ & $3.92 \mathrm{e}-02$ & $4.53 \mathrm{e}-02$ \\
10 & 1147 & $2.09 \mathrm{e}+00$ & $1.74 \mathrm{e}-01$ & $1.91 \mathrm{e}+00$ & $2.36 \mathrm{e}-02$ & $3.44 \mathrm{e}-02$ \\
12 & 2651 & $1.39 \mathrm{e}+00$ & $1.03 \mathrm{e}-01$ & $1.29 \mathrm{e}+00$ & $1.81 \mathrm{e}-02$ & $2.02 \mathrm{e}-02$ \\
14 & 6340 & $1.04 \mathrm{e}+00$ & $6.32 \mathrm{e}-02$ & $9.74 \mathrm{e}-01$ & $1.22 \mathrm{e}-02$ & $1.17 \mathrm{e}-02$ \\
\hline
\end{tabular}


TABLE 7.2

Example 1: Convergence history of the adaptive FEM, Part II: Components of the error estimator and data oscillations and consisteny error

\begin{tabular}{|r||c|c|c|c|c|c|}
\hline$\ell$ & $N_{\text {dof }}$ & $\eta_{y}$ & $\eta_{\bar{p}}$ & $o s c_{\ell}\left(u^{d}\right)$ & $o s c_{\ell}\left(y^{d}\right)$ & $\tilde{e}_{c}\left(u, u_{\ell}\right)$ \\
\hline 0 & 5 & $3.95 \mathrm{e}+01$ & $7.05 \mathrm{e}+00$ & $1.48 \mathrm{e}+01$ & $4.99 \mathrm{e}+00$ & $5.14 \mathrm{e}-02$ \\
1 & 13 & $2.19 \mathrm{e}+01$ & $2.04 \mathrm{e}+00$ & $1.37 \mathrm{e}+01$ & $5.42 \mathrm{e}-01$ & $0.00 \mathrm{e}+00$ \\
2 & 41 & $9.83 \mathrm{e}+00$ & $8.10 \mathrm{e}-01$ & $1.36 \mathrm{e}+01$ & $6.22 \mathrm{e}-01$ & $8.48 \mathrm{e}-02$ \\
4 & 105 & $3.67 \mathrm{e}+00$ & $4.35 \mathrm{e}-01$ & $9.42 \mathrm{e}+00$ & $3.32 \mathrm{e}-01$ & $0.00 \mathrm{e}+00$ \\
6 & 244 & $1.63 \mathrm{e}+00$ & $2.60 \mathrm{e}-01$ & $5.99 \mathrm{e}+00$ & $1.11 \mathrm{e}-01$ & $0.00 \mathrm{e}+00$ \\
8 & 532 & $1.17 \mathrm{e}+00$ & $1.69 \mathrm{e}-01$ & $3.17 \mathrm{e}+00$ & $4.47 \mathrm{e}-02$ & $0.00 \mathrm{e}+00$ \\
10 & 1147 & $7.72 \mathrm{e}-01$ & $1.22 \mathrm{e}-01$ & $1.90 \mathrm{e}+00$ & $2.17 \mathrm{e}-02$ & $0.00 \mathrm{e}+00$ \\
12 & 2651 & $4.71 \mathrm{e}-01$ & $7.37 \mathrm{e}-02$ & $1.29 \mathrm{e}+00$ & $9.27 \mathrm{e}-03$ & $0.00 \mathrm{e}+00$ \\
14 & 6340 & $2.93 \mathrm{e}-01$ & $4.55 \mathrm{e}-02$ & $9.74 \mathrm{e}-01$ & $4.62 \mathrm{e}-03$ & $0.00 \mathrm{e}+00$ \\
\hline
\end{tabular}

TABLE 7.3

Example 1: Convergence history of the adaptive FEM, Part III: Average values of the local estimators

\begin{tabular}{|r|r||c|c|c|c|c|c|}
\hline$\ell$ & $N_{\text {dof }}$ & $\eta_{y, T}$ & $\eta_{\bar{p}, T}$ & $\eta_{y, E}$ & $\eta_{\bar{p}, E}$ & $o s c_{T}\left(u^{d}\right)$ & $o s c_{T}\left(y^{d}\right)$ \\
\hline 0 & 5 & $1.88 \mathrm{e}+01$ & $2.32 \mathrm{e}+00$ & $6.05 \mathrm{e}+00$ & $2.65 \mathrm{e}+00$ & $7.40 \mathrm{e}+00$ & $2.49 \mathrm{e}+00$ \\
1 & 13 & $3.53 \mathrm{e}+00$ & $2.39 \mathrm{e}-01$ & $6.56 \mathrm{e}-01$ & $2.97 \mathrm{e}-01$ & $1.71 \mathrm{e}+00$ & $6.77 \mathrm{e}-02$ \\
2 & 41 & $6.11 \mathrm{e}-01$ & $6.26 \mathrm{e}-02$ & $3.13 \mathrm{e}-02$ & $1.66 \mathrm{e}-02$ & $7.57 \mathrm{e}-01$ & $2.61 \mathrm{e}-02$ \\
4 & 105 & $1.42 \mathrm{e}-01$ & $2.22 \mathrm{e}-02$ & $1.54 \mathrm{e}-02$ & $4.57 \mathrm{e}-03$ & $3.49 \mathrm{e}-01$ & $1.07 \mathrm{e}-02$ \\
6 & 244 & $3.84 \mathrm{e}-02$ & $8.12 \mathrm{e}-03$ & $4.70 \mathrm{e}-03$ & $1.43 \mathrm{e}-03$ & $1.37 \mathrm{e}-01$ & $2.28 \mathrm{e}-03$ \\
8 & 532 & $2.14 \mathrm{e}-02$ & $3.44 \mathrm{e}-03$ & $1.34 \mathrm{e}-03$ & $4.89 \mathrm{e}-04$ & $4.08 \mathrm{e}-02$ & $6.28 \mathrm{e}-04$ \\
10 & 1147 & $1.03 \mathrm{e}-02$ & $1.56 \mathrm{e}-03$ & $3.73 \mathrm{e}-04$ & $1.79 \mathrm{e}-04$ & $1.32 \mathrm{e}-02$ & $2.01 \mathrm{e}-04$ \\
12 & 2651 & $4.50 \mathrm{e}-03$ & $6.53 \mathrm{e}-04$ & $9.77 \mathrm{e}-05$ & $5.04 \mathrm{e}-05$ & $3.68 \mathrm{e}-03$ & $5.17 \mathrm{e}-05$ \\
14 & 6340 & $1.88 \mathrm{e}-03$ & $2.70 \mathrm{e}-04$ & $2.46 \mathrm{e}-05$ & $1.33 \mathrm{e}-05$ & $1.11 \mathrm{e}-03$ & $1.41 \mathrm{e}-05$ \\
\hline
\end{tabular}

TABLE 7.4

Example 1: Convergence history of the adaptive FEM, Part IV: Percentages of the bulk criterion

\begin{tabular}{|r|r||c|c|c|c|c|}
\hline$\ell$ & $N_{\text {dof }}$ & $M^{f b, T}$ & $M^{\eta, E}$ & $M^{\eta, T}$ & $M^{o s c_{1}, T}$ & $M^{o s c_{2}, T}$ \\
\hline 0 & 15 & 100.0 & 75.0 & 75.0 & 75.0 & 75.0 \\
1 & 13 & 100.0 & 20.0 & 18.8 & 18.8 & 18.8 \\
2 & 41 & 43.8 & 13.6 & 12.5 & 7.8 & 6.2 \\
4 & 105 & 22.2 & 4.7 & 16.7 & 13.9 & 8.3 \\
6 & 244 & 29.7 & 8.0 & 12.2 & 10.7 & 8.5 \\
8 & 532 & 16.0 & 8.1 & 16.1 & 4.2 & 6.5 \\
10 & 1147 & 11.6 & 8.3 & 19.8 & 1.4 & 4.0 \\
12 & 2651 & 8.7 & 10.0 & 25.9 & 0.1 & 2.0 \\
14 & 6340 & 5.1 & 11.0 & 28.3 & 0.1 & 1.2 \\
\hline
\end{tabular}

The second example which has been taken from [28] features a Lagrange multiplier in $\mathcal{M}(\Omega)=C^{*}(\bar{\Omega})$ and an adjoint state $p$ which is in $W^{1, s}(\Omega)$ for any $s \in(1,2)$.

Example 2: Lagrange multiplier in $C^{*}(\bar{\Omega})$

The data of the problem are as follows

$$
\begin{aligned}
& \Omega:=B(0,1) \quad, \quad \Gamma_{D}=\emptyset \quad, \quad y^{d}(r):=4+\frac{1}{\pi}-\frac{1}{4 \pi} r^{2}+\frac{1}{2 \pi} \ln (r), \\
& u^{d}(r):=4+\frac{1}{4 \pi} r^{2}-\frac{1}{2 \pi} \ln (r) \quad, \quad \alpha:=1.0 \quad, \quad c=1.0 \quad, \quad \psi(r):=r+4 .
\end{aligned}
$$

The optimal solution is given by:

$$
y(r) \equiv 4 \quad, \quad p(r)=\frac{1}{4 \pi} r^{2}-\frac{1}{2 \pi} \ln (r),
$$




$$
u(r) \equiv 4 \quad, \quad \sigma=\delta_{0}
$$
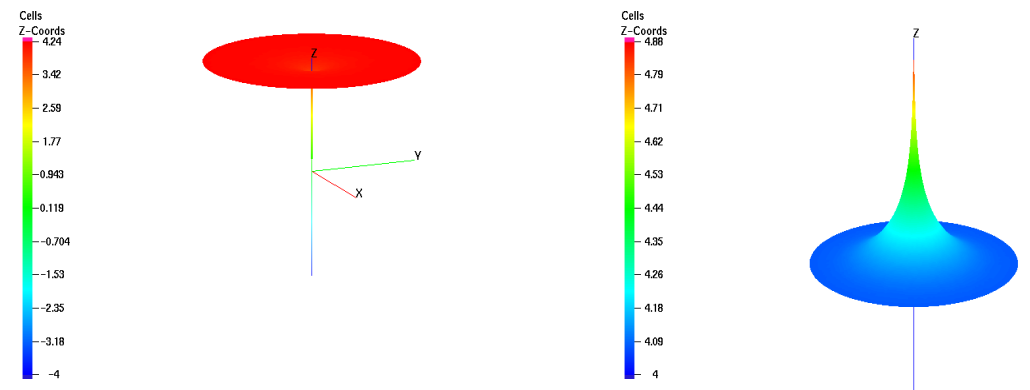

FIG. 7.6. Example 2: Visualization of the desired state $y^{d}$ (left) and the control shift $u^{d}$ (right)
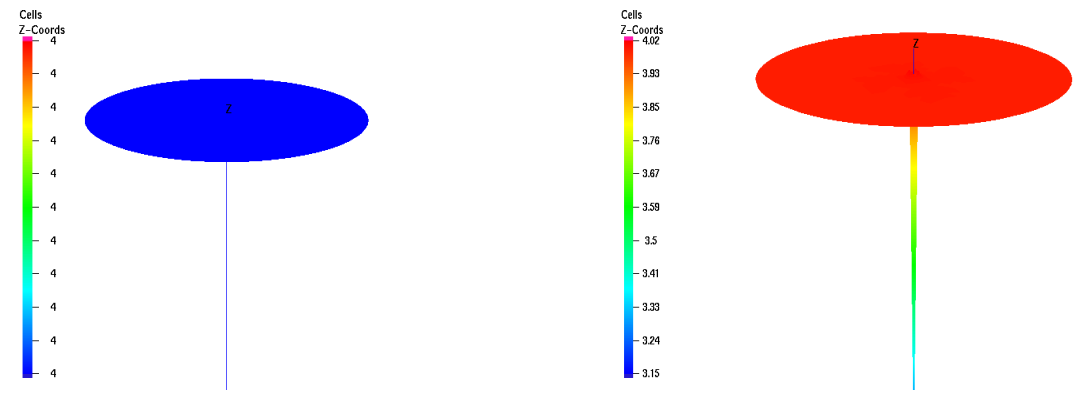

FIG. 7.7. Example 2: Visualization of the discrete state $y_{l}$ (left) and the discret control $u_{l}$ (right) on an adaptive generated mesh with 6735 nodes
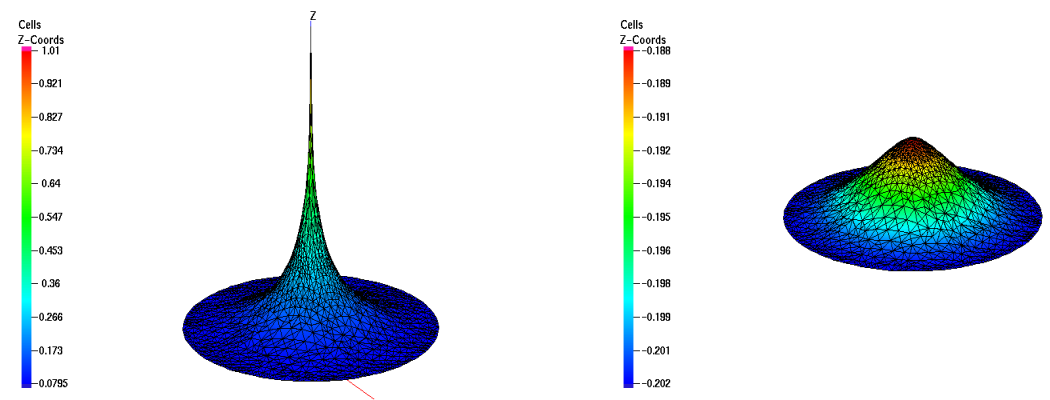

FIG. 7.8. Example 2: Visualization of the discrete adjoint state $p_{l}$ (left) and the discrete modified adjoint state $\bar{p}_{l}$ (right) on an adaptive generated mesh with 6735 nodes

Figures 7.6,7.7, and 7.8 show a visualization of the desired state $y^{d}$, the control shift $u^{d}$, discrete state $y_{\ell}$, the discrete control $u_{\ell}$, the discrete adjoint state $p_{\ell}$ and the discrete modified adjoint state $\bar{p}_{\ell}$ with respect to a simplicial triangulation consisting of 6735 nodal points. 

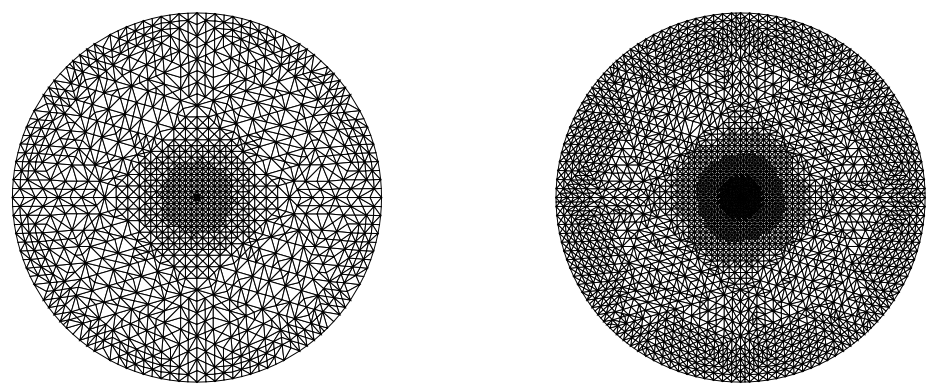

FIG. 7.9. Example 2: Adaptively generated grid after 12 (left) and 14 (right) refinement steps, $\Theta_{i}=0.7$

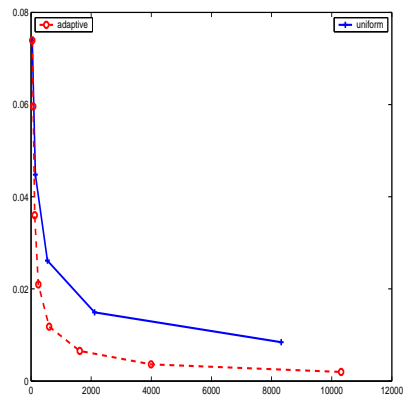

FIG. 7.10. Example 2: Adaptive versus uniform refinement, $\Theta_{i}=0.7$

The initial simplicial triangulation $\mathcal{T}_{h_{0}}$ has been chosen by means of the five nodal points $(0,0),(1,0),(0,1),(-1,0),(0,-1)$, resulting in five congruent triangles. During the refinement process each new point on a boundary edge has been projected onto $\partial B(0,1)$.

Table 4 - Table 8 contain the same data as in Example 1 documenting the history of the adaptive refinement process, whereas Figure 7.10 displays adaptive versus uniform refinement. In this example, the coincidence set consists of the single point $(0,0)^{T}$.

TABLE 7.5

Example 2: Convergence history of the adaptive FEM, Part I: Total discretization error and discretization errors in the state, control, adjoint state and modified adjoint state

\begin{tabular}{|r|r|c|c|c|c|c|}
\hline$\ell$ & $N_{\text {dof }}$ & $\left\|z-z_{\ell}\right\|$ & $\left\|y-y_{\ell}\right\|_{1}$ & $\left\|u-u_{\ell}\right\|_{0}$ & $\left\|p-p_{\ell}\right\|_{0}$ & $\left\|\bar{p}-\bar{p}_{\ell}\right\|_{1}$ \\
\hline 0 & 5 & $1.55 \mathrm{e}-01$ & $1.20 \mathrm{e}-02$ & $1.43 \mathrm{e}-01$ & $6.46 \mathrm{e}-02$ & $3.81 \mathrm{e}-02$ \\
1 & 13 & $1.13 \mathrm{e}-01$ & $8.51 \mathrm{e}-03$ & $1.04 \mathrm{e}-01$ & $3.73 \mathrm{e}-02$ & $1.74 \mathrm{e}-02$ \\
2 & 41 & $7.39 \mathrm{e}-02$ & $4.43 \mathrm{e}-03$ & $6.95 \mathrm{e}-02$ & $1.86 \mathrm{e}-02$ & $9.01 \mathrm{e}-03$ \\
4 & 73 & $5.96 \mathrm{e}-02$ & $2.30 \mathrm{e}-03$ & $5.73 \mathrm{e}-02$ & $1.00 \mathrm{e}-02$ & $7.36 \mathrm{e}-03$ \\
6 & 121 & $3.60 \mathrm{e}-02$ & $1.79 \mathrm{e}-03$ & $3.42 \mathrm{e}-02$ & $7.41 \mathrm{e}-03$ & $6.11 \mathrm{e}-03$ \\
8 & 243 & $2.10 \mathrm{e}-02$ & $1.07 \mathrm{e}-03$ & $1.99 \mathrm{e}-02$ & $4.13 \mathrm{e}-03$ & $4.02 \mathrm{e}-03$ \\
10 & 604 & $1.18 \mathrm{e}-02$ & $4.02 \mathrm{e}-04$ & $1.14 \mathrm{e}-02$ & $1.95 \mathrm{e}-03$ & $2.43 \mathrm{e}-03$ \\
12 & 1621 & $6.55 \mathrm{e}-03$ & $1.60 \mathrm{e}-04$ & $6.39 \mathrm{e}-03$ & $9.26 \mathrm{e}-04$ & $1.52 \mathrm{e}-03$ \\
14 & 3991 & $3.62 \mathrm{e}-03$ & $6.81 \mathrm{e}-05$ & $3.55 \mathrm{e}-03$ & $4.55 \mathrm{e}-04$ & $8.79 \mathrm{e}-04$ \\
\hline
\end{tabular}


TABLE 7.6

Example 2: Convergence history of the adaptive FEM, Part II: Components of the error estimator and the data oscillations and the consistency error

\begin{tabular}{|r|r||c|c|c|c|c|}
\hline$\ell$ & $N_{\text {dof }}$ & $\eta_{y}$ & $\eta_{\bar{p}}$ & osc $_{\ell}\left(u^{d}\right)$ & osc $_{\ell}\left(y^{d}\right)$ & $\tilde{e}_{c}\left(u, u_{l}\right)$ \\
\hline 0 & 5 & $1.91 \mathrm{e}-01$ & $1.38 \mathrm{e}-01$ & $1.73 \mathrm{e}-01$ & $1.36 \mathrm{e}-01$ & $0.00 \mathrm{e}+00$ \\
1 & 13 & $7.32 \mathrm{e}-02$ & $7.62 \mathrm{e}-02$ & $1.29 \mathrm{e}-01$ & $4.36 \mathrm{e}-02$ & $0.00 \mathrm{e}+00$ \\
2 & 41 & $2.45 \mathrm{e}-02$ & $3.83 \mathrm{e}-02$ & $8.14 \mathrm{e}-02$ & $1.26 \mathrm{e}-02$ & $0.00 \mathrm{e}+00$ \\
4 & 73 & $1.02 \mathrm{e}-02$ & $2.54 \mathrm{e}-02$ & $5.95 \mathrm{e}-02$ & $7.78 \mathrm{e}-03$ & $0.00 \mathrm{e}+00$ \\
6 & 121 & $3.11 \mathrm{e}-03$ & $1.97 \mathrm{e}-02$ & $3.56 \mathrm{e}-02$ & $4.96 \mathrm{e}-03$ & $0.00 \mathrm{e}+00$ \\
8 & 243 & $9.10 \mathrm{e}-04$ & $1.32 \mathrm{e}-02$ & $2.06 \mathrm{e}-02$ & $1.87 \mathrm{e}-03$ & $0.00 \mathrm{e}+00$ \\
10 & 604 & $2.59 \mathrm{e}-04$ & $8.07 \mathrm{e}-03$ & $1.17 \mathrm{e}-02$ & $8.27 \mathrm{e}-04$ & $0.00 \mathrm{e}+00$ \\
12 & 1621 & $7.22 \mathrm{e}-05$ & $4.75 \mathrm{e}-03$ & $6.54 \mathrm{e}-03$ & $3.16 \mathrm{e}-04$ & $0.00 \mathrm{e}+00$ \\
14 & 3991 & $2.01 \mathrm{e}-05$ & $2.89 \mathrm{e}-03$ & $3.62 \mathrm{e}-03$ & $1.41 \mathrm{e}-04$ & $0.00 \mathrm{e}+00$ \\
\hline
\end{tabular}

TABLE 7.7

Example 2: Convergence history of the adaptive FEM, Part III: Average values of the local estimators

\begin{tabular}{|r|r||c|c|c|c|c|c|}
\hline$\ell$ & $N_{\text {dof }}$ & $\eta_{y, T}$ & $\eta_{\bar{p}, T}$ & $\eta_{y, E}$ & $\eta_{\bar{p}, E}$ & $o s c_{\ell}\left(u^{d}\right)$ & $o s c_{\ell}\left(y^{d}\right)$ \\
\hline 0 & 5 & $9.95 \mathrm{e}-02$ & $6.73 \mathrm{e}-02$ & $8.01 \mathrm{e}-03$ & $1.43 \mathrm{e}-02$ & $8.67 \mathrm{e}-02$ & $6.80 \mathrm{e}-02$ \\
1 & 13 & $1.03 \mathrm{e}-02$ & $1.58 \mathrm{e}-02$ & $1.01 \mathrm{e}-03$ & $3.21 \mathrm{e}-03$ & $1.91 \mathrm{e}-02$ & $8.67 \mathrm{e}-03$ \\
2 & 41 & $9.39 \mathrm{e}-04$ & $3.61 \mathrm{e}-03$ & $8.46 \mathrm{e}-05$ & $6.85 \mathrm{e}-04$ & $3.39 \mathrm{e}-03$ & $1.08 \mathrm{e}-03$ \\
4 & 73 & $3.20 \mathrm{e}-04$ & $1.94 \mathrm{e}-03$ & $1.99 \mathrm{e}-05$ & $3.14 \mathrm{e}-04$ & $1.75 \mathrm{e}-03$ & $4.88 \mathrm{e}-04$ \\
6 & 121 & $8.10 \mathrm{e}-05$ & $1.19 \mathrm{e}-03$ & $8.36 \mathrm{e}-06$ & $1.74 \mathrm{e}-04$ & $7.40 \mathrm{e}-04$ & $2.49 \mathrm{e}-04$ \\
8 & 243 & $1.44 \mathrm{e}-05$ & $5.36 \mathrm{e}-04$ & $1.33 \mathrm{e}-06$ & $5.34 \mathrm{e}-05$ & $2.16 \mathrm{e}-04$ & $7.00 \mathrm{e}-05$ \\
10 & 604 & $2.20 \mathrm{e}-06$ & $2.15 \mathrm{e}-04$ & $1.37 \mathrm{e}-07$ & $1.44 \mathrm{e}-05$ & $5.28 \mathrm{e}-05$ & $1.85 \mathrm{e}-05$ \\
12 & 1621 & $2.80 \mathrm{e}-07$ & $7.80 \mathrm{e}-05$ & $1.24 \mathrm{e}-08$ & $3.36 \mathrm{e}-06$ & $1.15 \mathrm{e}-05$ & $4.07 \mathrm{e}-06$ \\
14 & 3991 & $4.32 \mathrm{e}-08$ & $3.09 \mathrm{e}-05$ & $1.31 \mathrm{e}-09$ & $8.29 \mathrm{e}-07$ & $2.76 \mathrm{e}-06$ & $1.04 \mathrm{e}-06$ \\
\hline
\end{tabular}

TABLE 7.8

Example 2: Convergence history of the adaptive FEM, Part IV: Percentages in the bulk criteria

\begin{tabular}{|r|r||c|c|c|c|c|}
\hline$\ell$ & $N_{\text {dof }}$ & $M^{f b, T}$ & $M^{\eta, E}$ & $M^{\eta, T}$ & $M^{o s c_{1}, T}$ & $M^{o s c_{2}, T}$ \\
\hline 0 & 5 & 100.0 & 75.0 & 75.0 & 75.0 & 75.0 \\
1 & 13 & 25.0 & 30.0 & 25.0 & 31.2 & 25.0 \\
2 & 41 & 6.3 & 27.3 & 7.8 & 6.2 & 12.5 \\
4 & 73 & 6.5 & 10.8 & 25.8 & 5.6 & 17.7 \\
6 & 121 & 3.9 & 7.5 & 30.0 & 3.4 & 16.4 \\
8 & 243 & 1.8 & 11.3 & 31.7 & 1.6 & 21.4 \\
10 & 604 & 0.7 & 15.2 & 37.9 & 0.5 & 17.1 \\
12 & 1621 & 0.3 & 8.1 & 39.8 & 0.2 & 15.9 \\
14 & 3991 & 0.1 & 8.7 & 47.4 & 0.1 & 9.8 \\
\hline
\end{tabular}

\section{REFERENCES}

[1] M. Ainsworth and J.T. Oden (2000). A Posteriori Error Estimation in Finite Element Analysis. Wiley, Chichester.

[2] I. Babuska And T. Strouboulis (2001). The Finite Element Method and its Reliability. Clarendon Press, Oxford.

[3] W. Bangerth and R. Rannacher (2003). Adaptive Finite Element Methods for Differential Equations. Lectures in Mathematics. ETH-Zürich. Birkhäuser, Basel.

[4] R. Becker, H. Kapp, and R. Rannacher (2000). Adaptive finite element methods for optimal control of partial differential equations: Basic concepts. SIAM J. Control Optim. 39, 113132 .

[5] M. Bergounioux, K. Ito, and K. Kunisch (1999). Primal-dual strategy for constrained optimal control problems. SIAM J. Control and Optimization 34, 1176-1194 
[6] M. Bergounioux, M. Haddou, M. Hintermüller, and K. Kunisch (2000). A comparison of a Moreau-Yosida based active set strategy and interior point methods for constrained optimal control problems. SIAM J. Optim. 11, 495-521.

[7] M. Bergounioux And K. Kunisch (2002). On the structure of the Lagrange multiplier for state-constrained optimal control problems. Systems and Control Letters 48, 169-176.

[8] E. CASAS (1986). Control of an elliptic problem with pointwise state controls. SIAM J. Control Optim. 24, 1309-1318.

[9] E. Casas And M. Mateos (2002). Uniform convergence of the FEM applications to state constrained control problems. Comp. Appl. Math. 21, 67-100.

[10] K. Deckelnick And M. Hinze (2006). Convergence of a finite element approximation to a state constrained elliptic control problem. Preprint, Dept. of Mathematics, Dresden University of Technology.

[11] W. DöRfler (1996). A convergent adaptive algorithm for Poisson's equation. SIAM J. Numer. Anal. 33, 1106-1124.

[12] K. Eriksson, D. Estep, P. Hansbo, and C. Johnson (1995). Computational Differential Equations. Cambridge University Press, Cambridge.

[13] F. FALK (1973). Approximation of a class of optimal control problems with order of convergence estimates. J. Math. Anal. Appl. 44, 28-47.

[14] A. Gaevskaya, R.H.W. Hoppe, Y. Iliash, And M. Kieweg (2006). A posteriori error analysis of control constrained distributed and boundary control problems. Proc. Conf. Advances in Scientific Computing, Moscow, Russia (O. Pironneau et al.; eds.), Russian Academy of Sciences, Moscow.

[15] A. Gaevskaya, R.H.W. Hoppe, Y. Iliash, and M. Kieweg (2007). Convergence analysis of an adaptive finite element method for distributed control problems with control constraints. Proc. Conf. Optimal Control for PDEs, Oberwolfach, Germany (G. Leugering et al.; eds.), Birkhäuser, Basel.

[16] A. Gaevskaya, R.H.W. Hoppe, and S. Repin (2006). A Posteriori Estimates for Cost Functionals of Optimal Control Problems. In: Numerical Mathematics and Advanced Applications (A. Bermudez de Castro et al.; eds.), pp. 308-316, Springer, Berlin-Heidelberg-New York.

[17] P. Grisvard (1985). Elliptic Problems in Nonsmooth Domains. Pitman, Boston.

[18] M. Hintermüller, R.H.W. Hoppe, Y. Iliash, and M. Kieweg (2007). An a posteriori error analysis of adaptive finite element methods for distributed elliptic control problems with control constraints. to appear in ESAIM, COCV.

[19] M. Hintermüller And K. KUnisCh (2006). Feasible and non-interior path-following in constrained minimization with low multiplier regularity. SIAM J. Control Optim., 45, 11981221.

[20] M. Hintermüller AND K. Kunisch (2006). Stationary state constrained optimal control problems. Preprint IFB-Report No. 3, Karl-Franzens-University of Graz.

[21] M. Hintermüller And K. Kunisch (2007). Path-following methods for a class of constrained minimization problems in function space. to appear in SIAM J. Optimization.

[22] R.H.W. Hoppe, Y. Iliash, C. Iyyunni, and N. Sweilam (2006). A posteriori error estimates for adaptive finite element discretizations of boundary control problems. J. Numer. Anal. 14, 57-82.

[23] K. Kunisch And A. Rösch (2002). Primal-dual active set strategy for a general class of constrained optimal control problems. SIAM J. Control and Optimization 42, 321-334.

[24] R. Li, W. LiU, H. Ma, AND T. TANG (2002). Adaptive finite element approximation for distributed elliptic optimal control problems. SIAM J. Control Optim. 41, 1321-1349.

[25] J.L. Lions (1971). Optimal Control of Systems Governed by Partial Differential Equations. Springer, Berlin-Heidelberg-New York.

[26] W. LiU AND N. YAN (2001). A posteriori error estimates for distributed optimal control problems. Adv. Comp. Math. 15, 285-309.

[27] W. LiU AND N. YAN (2003). A posteriori error estimates for convex boundary control problems. Preprint, Institute of Mathematics and Statistics, University of Kent, Canterbury.

[28] C. Meyer, U. Prüfert, and F. Tröltzssch (2005). On two numerical methods for stateconstrained elliptic control problems. Preprint, Dept. of Mathematics, Berlin University of Technology.

[29] P. Morin, R.H. Nochetto, and K.G. Siebert (2000). Data oscillation and convergence of adaptive FEM. SIAM J. Numer. Anal., 38, 2, 466-488.

[30] P. NeittaAnmäki and S. Repin (2004). Reliable methods for mathematical modelling. Error control and a posteriori estimates. Elsevier, New York.

[31] F. Tröltzsch (2005). Optimale Steuerung partieller Differentialgleichungen. Vieweg, Wies- 
baden, 2005

[32] R. Verfürth (1996). A Review of A Posteriori Estimation and Adaptive Mesh-Refinement Techniques. Wiley-Teubner, New York, Stuttgart. 